\title{
Mise en évidence d'une phase tectonique au Santonien du versant Nord du Haut Atlas Occidental, Maroc
}

\author{
Ahmed Algouti Pes \\ Abdellah Algouti Pes \\ Fatiha Hadach Phd \\ University Cadi Ayyad, Faculty of Sciences Semlalia , \\ Laboratory Sedimentary Basins Geology of Moroccan "GEOBASSMA" \\ Geology Department. Marrakech, Morocco
}

doi: 10.19044/esj.2016.v12n3p107 URL:http://dx.doi.org/10.19044/esj.2016.v12n3p107

\begin{abstract}
At the level of the north hillside of the western High Atlas, only the training of limestones and dolomitic marls of Aït Abbes was recognized in Senonian. It is topped by the phosphated series.

To highlight a tectonic action at the end of lower Santonian of Sidi Bou Othman's region and which is probably due to a halocinique phase, three cuts were been lifted on both sides of the Assif Aït Tabgaw.

This tectonic phase appears by angular unconformity within the deposits of the training of Aït Abbes, microfaults, synsedimentary slidings, and monogenic breaches...

It also structures the whole region at the high bottom or emergent low lands forming several small basins confined and supersaturated in brines. Then, following an important peneplanation, a platform of sebkha type is set up on the whole region. Some brief marine incursions reach the eastern basins.
\end{abstract}

Keywords: Santonien, Halocinique phase, Sedimentology, Eustatism, Unconformity

\section{Resume}

Au niveau du versant Nord du Haut Atlas occidental seule la formation des calcaires et marnes dolomitiques d'Aït Abbes a été reconnue au Sénonien. Elle est surmontée par la série phosphatée.

Pour mettre en évidence une action tectonique à la fin du Santonien inférieur de la région de Sidi Bou Othman et qui est due probablement à une phase halocinique, trois coupes sériées ont été relevées de part et d'autre de l'Assif Aït Tabgaw.

Cette phase tectonique se présente par des discordances angulaires au sein 
des dépôts de la formation d'Aït Abbes, des microfailles et glissements synsédimentaires, des brèches monogéniques...

Elle structure aussi l'ensemble de la région en haut fonds ou en terres émergées peu élevées formant plusieurs petits bassins confinés et sursaturés en saumures. Puis, à la suite d'une pénéplanation importante, une plateforme de type sebkha se met en place sur l'ensemble de la région. Quelques brèves incursions marines atteignent les bassins orientaux.

Mots clés : Santonien, Phase halocinique, Sédimentologie, Eustatisme, Discordances

\section{Introduction}

Notre secteur d'étude se situe au versant Nord du Haut Atlas Occidental, à une quarantaine de kilomètres au SW d'Amizmiz (Fig.1), en prenant la piste passant par la mine de Sidi Bou Othman. Trois coupes sériées ont été levées, orientées Est-Ouest (Fig.2), afin de mettre en évidence les variations latérales de lithofaciès et d'épaisseur, que connaissent les dépôts Sénoniens de ce secteur. La première coupe se situe à la rive droite de l'Assif Aït Tabgaw, près du douar Tagadirt. La troisième coupe se localise à la rive gauche du même Assif (Planche1 / Photo 1). La deuxième coupe, se situe entre les deux autres coupes, au niveau de l'Assif Aït Tabgaw. Afin d'éviter des répétitions fastidieuses, seule la première coupe va être décrite en détail, tout en notant les différentes variations latérales rencontrées en se déplaçant à l'Ouest.
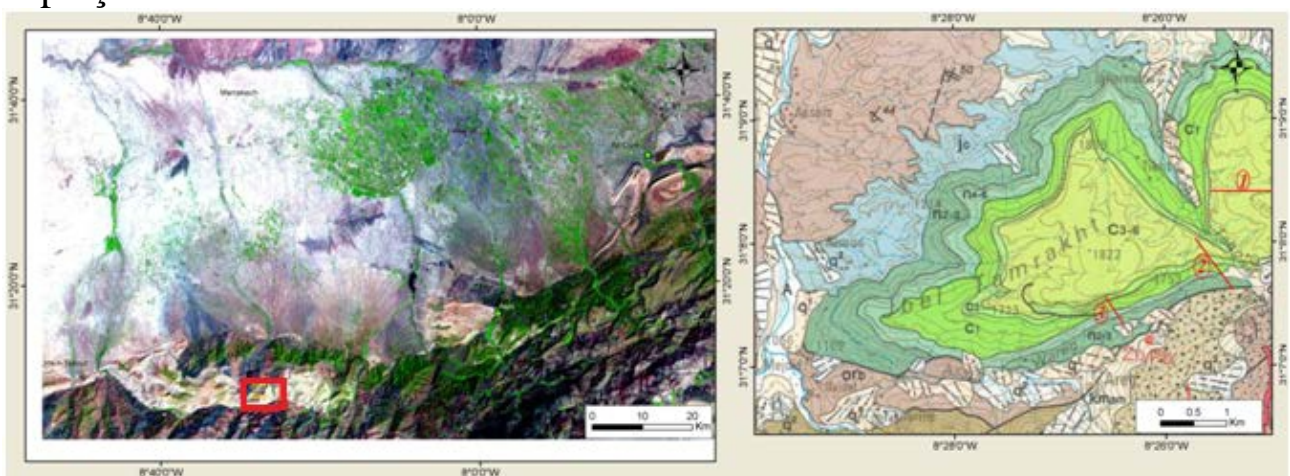

Fig1 : A : Situation géographique de de Sidi Bou Othman / B : Localisation des Trois coupes $(1,2,3)$

L'étude des terrains sénoniens du Haut Atlas Occidental, Maroc (Algouti,1999, Hadach et al., 2015) a permis d'individualiser :

la formation des calcaires et marnes dolomitiques d'Anou-Nfeg, la formation des calcaires et marnes dolomitiques d'Aït Abbès, la formation des marnes de l'Oued Lahouar et la série phosphatée (formation des calcaires du Tagragra et la formation des sables phosphatée de 
Chichaoua), qui sont d'âge respectivement Coniacien, Santonien, Campanien, Maastrichtien.

La formation des calcaires et marnes dolomitiques d'Anou-Nfeg, rapportée au Coniacien par les ammonites, les bryozoaires et les associations d'ostracodes.

La formation des calcaires et marnes dolomitiques d'Aït Abbès, datée du Santonien par des bryozoaires, des associations d'ostracodes, des échinides et des ammonites.

La formation des marnes de l’Oued Lahouar datée du Campanien, essentiellement par les ostracodes.

La série phosphatée, attribuée au Maastrichtien par une faune d'ostracodes, oursins, dents de poissons et d'ammonites.

Le Sénonien de cette région est délimité à sa base par la corniche cénomano-turonienne et à son sommet par une faille inverse qui le met en contact avec le socle (Planche1/ Photo 2).
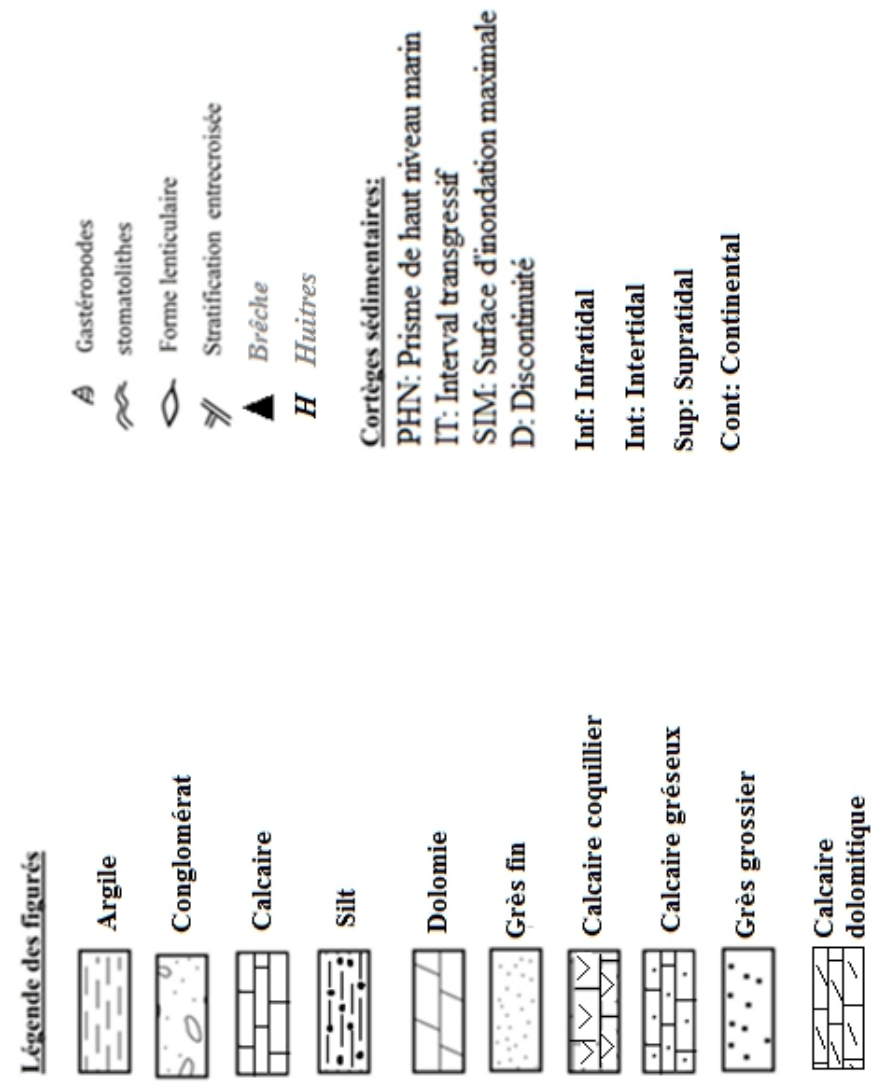


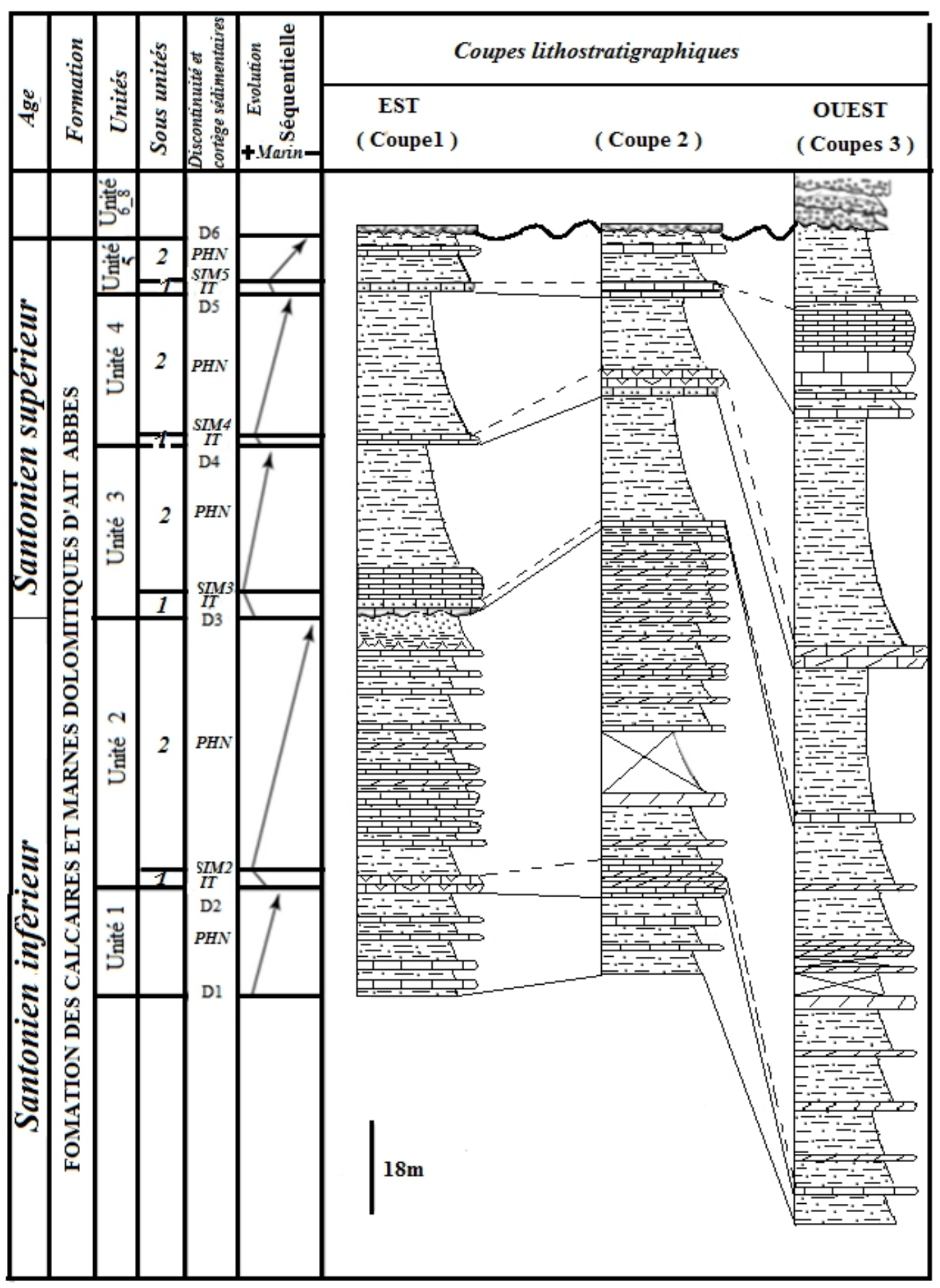

Fig.2 : Corrélation des 3 coupes lithostratigraphiques du secteur de Sidi Bou Othman. 


\section{II : formation des calcaires et marnes dolomitiques d'ait abbes}

Elle est délimitée par les discontinuités D1 et D6, et est subdivisée en 5 unités: U1, U2, U3, U4 et U5.

\section{Unité 1}

C'est une unité de 19m d'épaisseur, elle repose sur le Turonien supérieur, lequel montre à sa surface une discontinuité D1 matérialisée par une ferruginisation ((Planche1/ Photo 3). Le microfaciès de cette dernière est une dolomicrite ferrugineuse mudstone et vacuolaire à sheet cracks. Les vacuoles de dissolution sont comblées partiellement par la sparite et tapissées par des rhomboèdres dolomitiques, parfois on note la présence de silt vadose.

Tous ces caractères attestent d'une mise à l'air libre, à la fin du Turonien supérieur dans un milieu marin peu profond à énergie hydrodynamique faible.

Les dépôts Sénoniens qui suivent s'agencent de telle manière qu'on a à la base une dominance de bancs carbonatés généralement à aspect laminés avec parfois des brèches monogéniques de dissolution ((Planche1/ Photo 4). Ils montrent au microscope une dolomicrite à dolomicrosparite avec une disposition en une succession de petites lamines micritique ondulées d'origine algaire, avec des vacuoles de dissolution et des fenestrés qui sont comblées partiellement par la calcite drusique parfois à sédiment interne.

Au sommet on a une dominance de silts rouges, azoïques par rapport aux bancs carbonatés.

L'agencement vertical des faciès de cette unité traduit une baisse bathymétrique avec passage d'un milieu intertidal à supratidal dans une plate-forme interne à énergie hydrodynamique faible. Il s'agit d'un PHN.

En se déplaçant à l'Ouest, on a une diminution nette de l'épaisseur qui peut atteindre son minimum dans la troisième coupe où l'unité est formée uniquement par 3m de marne azoïque.

\section{Unité 2}

Elle est subdivisée en deux sous unité

\section{Sous unité 1}

C’est une sous unité carbonatée de $4 \mathrm{~m}$ d'épaisseur. Organisée en corps lenticulaires progradant vers le SE (Planche 2/ Photo 1) qui passent au sommet à des dépôts très fossilifères.

Les microfaciès des bancs ont donné de bas en haut.

$>$ Dolomicrite mudstone à rares petits grains de quartz (1\%);

$>$ Biodolomicrite wackestone. La biophase est constituée par des 
Foraminifères

planctoniques, nombreux Lamellibranches, Ostracodes, Echinodermes, Bryozoaires et Algues en éventail. Ceci est associé à quelques rares grains de quartz (1 à 2\%) et à des rhomboèdres dolomitiques zonés. Les éléments figurés s'organisent en petites lamines frustes.

L'organisation des faciès de cette sous unité marque un certain approfondissement du milieu (infralittoral) dans une plate-forme interne à communication momentanée avec le large. Il s’agit d’un interval transgressif qui se termine par un Sim (Sim2).

En passant à l'Ouest, on dénote un changement d'épaisseur et de faciès, ainsi on a :

- dans la deuxième coupe, une épaisseur qui peut atteindre 6,2m avec des dépôts formés par des bancs, généralement, bioturbés et fossilifère au sommet, qui se terminent par une surface oxydée;

- dans la troisième coupe, la sous unité se réduit à 1,5m d'épaisseur, avec une progradation des corps carbonatés de l'Est vers l'Ouest.

\section{Sous unité 2}

C'est une sous unité épaisse de 51m, formée par une alternance de carbonates et de silt, intercalés parfois au sommet par des grès.

Les carbonates de la base s'organisent en deux faciès :

- Le premier est un calcaire massif centimétrique généralement fossilifère à Huîtres et Gastéropodes, avec parfois des lamines parallèles et d'autres stromatolithiques vues au centre de la sous unité.

- Le deuxième est sous forme de plaquettes (10 à 20cm) non fossilifères montrant au microscope une dolomicrosparite ferrugineuse à sheet cracks et à traces de racines comblées par une dolomite granulaire.

Les carbonates du sommet sont généralement bréchifiés et laminés devenant de plus en plus gréseux verticalement.

Les silts sont rougeâtres, azoïques, plus abondants au sommet où ils deviennent légèrement gypsifères.

Les grès sont peu abondants à la base où ils s'organisent en petits microchenaux granodécroissant, et deviennent relativement fréquent au sommet, où ils sont affectés par des failles synsédimentaires et par une belle discordance angulaire (Planche 2/ Photo 2)

\section{Séquence élémentaire de la sous unité 2 de L'unité 2.}

C'est une séquence élémentaire (Fig 3) formée par trois termes. Nous avons de bas en haut:

- Terme A: Calcaire massif fossilifère à stromatolithes, caractérisant un milieu intertidal.

- Terme B: Plaquettes dolomitiques, brèchifiées, azoïques à traces 
de racines et à sheet cracks, traduisant une exondation du milieu de dépôt et mise à l'air libre. continentale.

- Terme C: Silt azoïque rouge à microchenaux gréseux d'origine

C'est une séquence granocroissante de comblement, qui voit augmenter les apports détritiques continentaux au profit des carbonates marins intertidaux.

L'organisation verticale des faciès, avec abondance des termes détritiques (Fig.3, termes $\mathrm{C}$ et $\mathrm{B}$ ) au profit du terme carbonaté (Fig.3, terme $\mathrm{A}$ ) montre un comblement dû à une chute bathymétrique, traduisant un PHN.

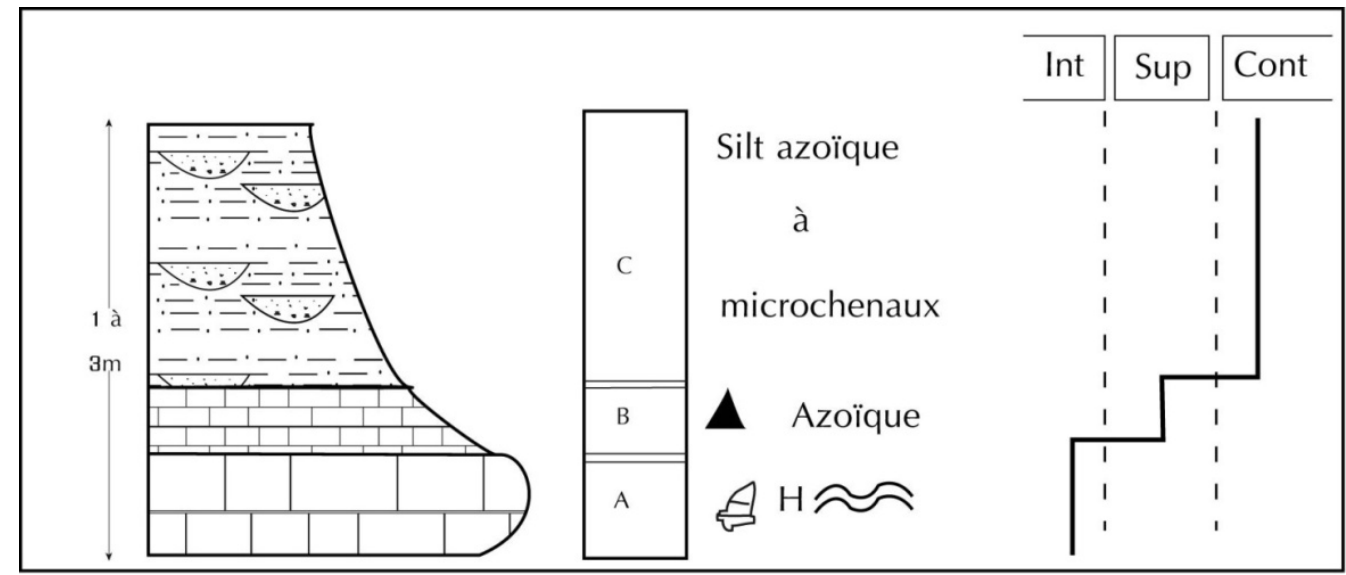

Fig 3 : Séquence élémentaire de la sous unité 2 de l'unité U2

\section{Comparaison avec le secteur ouest}

Les dépôts de cette sous unité montrent dans le secteur Ouest une abondance d'interbancs silteux qui peuvent atteindre 10m d'épaisseur, par rapport aux bancs carbonatés, ces derniers sont généralement dolomitiques laminés, non fossilifères, montrant rarement des petits dômes de stromatolithes, et de nombreux fenestrae dans la partie sommitale.

Unité $3(33 m)$

Elle est subdivisée en deux sous unités

\section{Sous unité 1}

C’est une sous unité carbonaté d'épaisseur réduite ( $2 \mathrm{~m})$. Sa base est ravinante et sa surface est fortement bioturbée et fossilifère Le microfaciès est une micrite dolomitique gréseuse (Planche 2 / Photo 3). La fraction silico-clastique est constituée par 15\% de grains de Quartz, à forme arrondie et à classement moyen. Ceci est associé à quelques fragments micritiques et quartziques et quelques grains phosphatés. La biophases est formée par des 
baguettes bioclastiques silicifiées. Cette Sous unité se réduit à l'Ouest en un banc centimétrique. La base ravinante indique un milieu très agité. Le sommet très bioturbé et fossilifère suggère un milieu propice à l'épanouissement organique. L'evolution verticale des faciès indique clairement un certain approfondissement du milieu dans une zone infralittorale, traduisant un IT qui se termine par un Sim (Sim3).

\section{Sous unité 2 (31m)}

Elle est formée par deux ensembles :

- Le premier $(7 \mathrm{~m})$ est carbonaté et est formé par des calcaires dolomitiques et parfois par des grès carbonatés. Les niveaux carbonatés du sommet montrent des structures en "tapis", des brèches monogéniques de remaniement et des trous de dissolution.

- Le deuxième ensemble est constitué par $24 \mathrm{~m}$ de silt rougeâtre et azoïque et (Planche 2 / Photo 4).

En se déplaçant à l'Ouest seul l'ensemble silteux azoïque est présent et est relativement épais (30m)

Le milieu de dépôt des bancs carbonatée de la base du secteur Est correspond à un milieu peu profond à tendance à l'émersion. En effet, les structures en "tipis » sont décrites dans certaines milieux actuels dans des zones intertidales à supertidales (BURRI et Al., 1973 ; PURSER, 1980). D'après Du DRESNAY (1976) ces structures sont formées en bordure du domaine maritime dans une zone temporairement émergée sous un climat chaud et aride.

\section{Unité 4}

Elle est subdivisée en deux sous unités.

\section{Sous unité 1}

C’est une sous unité très réduite $(2 \mathrm{~m})$ pouvant atteindre $5 \mathrm{~m}$ en allant à l'Ouest. Elle est constituée par un banc carbonaté, fossilifère au sommet à Lamellibranche notamment des Ostracodes, et se termine par une surface de discontinuité à nodules de silex (Planche 2 / Photo 5). Au microscope, il s'agit d'une dolomicrosparite à dolosparite à baguettes bioclastique silicifiées.

En allant vers l'Ouest, l'épaisseur devient de plus en plus importante. Cette sous unité est constituée dans se secteur par un banc lenticulaire montrant à sa base un calcaire graveleux, parfois à quartzite, d'aspect lumachéllique à Gastéropodes (Planche 3 / Photo 1 et 2), et au sommet une richesse en faune accompagnée d'une bioturbation intense et de stratification entrecroisée. Des microchenaux d'aspect lumachéllique, ont été notés, avec une progradation du NNE vers le SSW la surface sommitale de 
ce bans montre une discontinuité représentée par une croûte de 5 à 10 cm, très oxydée (Planche 3 / Photo 3 et 4 ).

La séquence élémentaire représentative de cette sous unité est formée à la base par un calcaire graveleux lenticulaire à tendance lumachéllique et à stratifications entrecroisées à direction NNE vers le SSW et au sommet par un niveau fin bioturbé montrant à son sommet des figures d'échappement d'eau. (Fig 4)

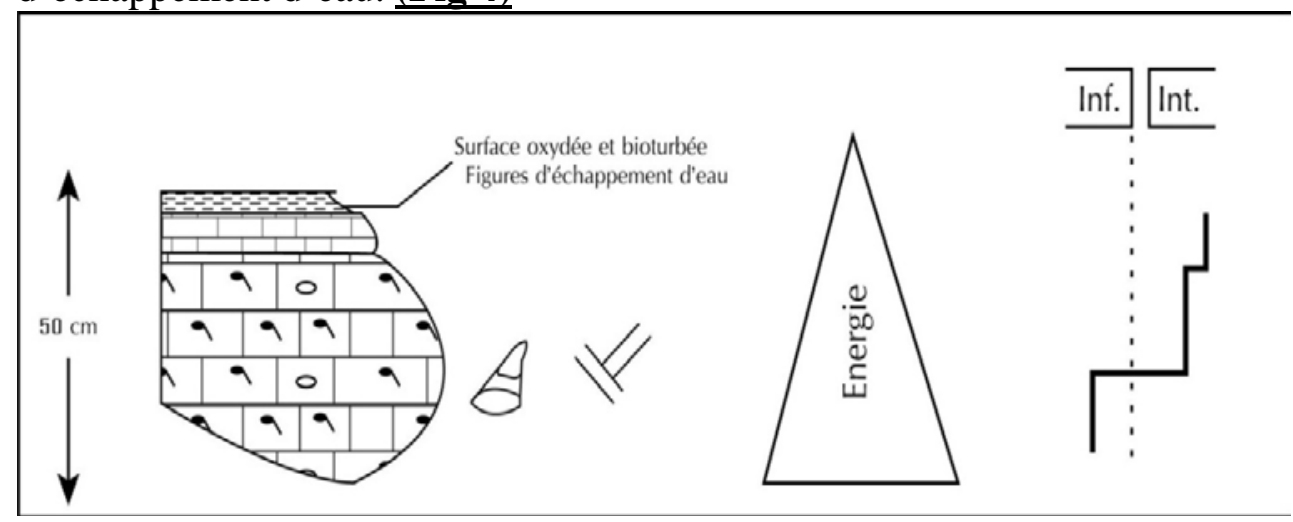

Fig 4: Séquence élémentaire représentative de la sous unité 1 de U4

L'analyse microscopique a donné une roche totalement recristallisée en calcite sparitique de telle manière qu'il est devenu difficile de mettre en évidence les éléments figurés existants. Les bioclastes ont été dissouts et comblés par la suite par la calcite granulaire ou drusique.

\section{Sous unité 2}

C'est une sous unité représentée uniquement par un dépôt de 30m de silts azoïques. En se déplaçant à l'Ouest, elle devient réduite dans la deuxième coupe où elle atteint $14 \mathrm{~m}$ et redevient encore épaisse dans la troisième coupe $(46 \mathrm{~m})$. L'aspect azoïque de ces dépôts suggère un milieu très peu profond à conditions défavorables à l'épanouissement d'organismes vivants.

\section{Unité 5}

Elle est subdivisée en deux sous unités.

\section{Sous unité 1}

Elle est d'épaisseur réduite $(2 \mathrm{~m})$ à l'Est ( $1^{\text {ère }}$ coupe), formée par des bancs carbonatés d'aspect stratocroissant. Leur microfaciès est une dolomicrite à dolomicrosparite légèrement gréseuse à silteuse avec 1 à 5\% de grains de quartz corrodés et arrondis. La texture est de type mudstone à wackestone. La biophase est constituée par des baguettes bioclastiques silicifiées. 
Dans le secteur ouest ( $3^{\text {ème }}$ coupe), cette sous unité est plus développée (22m). Elle est formée par des calcaires gréseux intertidaux devenant plus fossilifères au sommet avec notamment la présence des huîtres. Ces bancs calcaires alternent parfois avec des niveaux marneux centimétriques, surtout à la base.

Elle se termine par une surface ferrugineuse pleine de Lamellibranches. Il s'agit d'une biosparite silteuse à bioclastes phosphatés (SIM5).

\section{Sous unité 2}

Elle est formée à l'Est ( $1^{\text {ère }}$ coupe) par des silts évaporitiques azoïques rougeâtres, et à l'Ouest par des marnes jaunâtres azoïques intercalées à la base par un banc de calcaire fossilifère à Lamellibranche. Les bancs carbonatés sont formés dans un milieu marin calme peu profond (intertidal). Les interbancs caractérisent un milieu très peu profond (supratidal) à tendance au confinement.

\section{Série phosphatée}

Cette série débute par une base érosive. Elle est amputée au sommet par la faille inverse F1 qui le met en contact avec le socle. Donc seule une partie de cette formation est visible. Elle est formée par une succession de trois niveaux conglomératiques, azoïques, d'aspect granodécroissant, de forme lenticulaire, à base érosive et à matrice silteuse abondante. Ils traduisent le comblement de chenaux.

L'organisation verticale de ces trois niveaux traduit une granocroissance. Les galets sont émoussés ayant subit un léger transport, ils sont formés essentiellement par les sédiments sous-jacents.

\section{Conclusion}

Les trois coupes relevées de part et d'autre de l'Assif Aït Tabgaw, confirment le fonctionnement d'une phase halocinique à la fin du Santonien inférieur favorisée par la présence de failles majeures.

Ceci est dégagé à partir de plusieurs observations :

Avant l'intervention de cette phase (pendant le Santonien inférieur : les milieux et les épaisseurs sont relativement les mêmes sur tout le secteur d'étude, par contre après le jeu de la montée diapirique nous avons une variation d'épaisseur avec dans les secteurs Est et Ouest une épaisse série subsidente, et au niveau de l'Assif Aït Tabgaw l'épaisseur est très réduite ;

La mesure des paléocourants des corps sédimentaires de part et d'autre de l'Assif a donnée pour le secteur Est un sens NW vers le SE, et pour le secteur Ouest un paléocourant cheminant vers le SSW (Fig.5). 
La mesure de la direction et du pendage des failles synsédimentaires a donnée pour le secteur Oriental un sens SSE et pour le secteur Occidental un sens NNE (Fig.5).

La présence de discordance angulaire entre le Santonien inférieur et supérieur qui coïncide avec celle vue au niveau de la cuvette de Jbel Sour près d'Aït Ourir au versant Nord du Haut Atlas de Marrakech (Hadach et al., 2015).

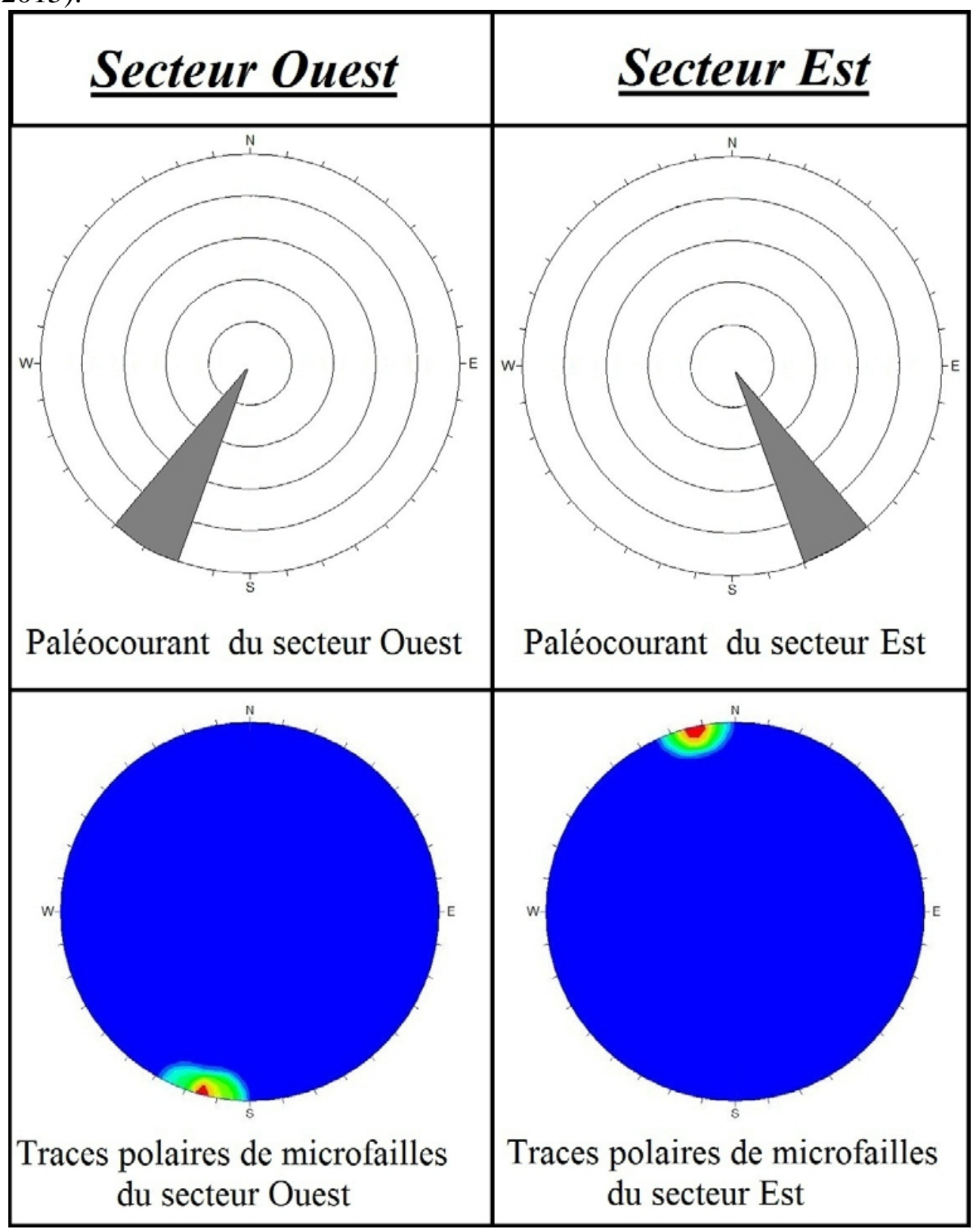

Fig.5 : Paléocourants et traces polaires de microfailles de la région de Sidi Bou Othman 


\section{Planche1}

Photo 1: vue panoramique du secteur Ouest (3ème coupe), montrant un Sénonien compris entre la corniche cénomano-turonienne (CT) et la faille inverse F1 qui le met en contact avec le socle.

Photo 2 : vue panoramique du secteur Est (1ère coupe), à côté de Douar Tagadirt, montrant une partie de la formation d'Ait Abbès.

Photo 3: discontinuité matérialisée par une surface oxydée, terminant la barre cénomano-turonienne.

Photo 4 : Séquence élémentaire de la sous unité 1 de l'unité U1. Elle est formée par 3 termes.

A : marne verdâtre a jaunâtre.

$\mathrm{B}$ : Calcaire à brèche monogénique de bioturbation.

$\mathrm{C}$ : Banc massif de calcaire dolomitique.

\section{Planche 2}

Photo 1: La sous unité 1 carbonatée, organisée en corps lenticulaires progradant vers le SE.

Photo 2: Discordance angulaire vue au sommet de U2 surmontée par un banc carbonaté à base érosive.

Photo 3: A) Banc massif carbonaté (sous unité 1 de U3) à base érodant les niveaux gréseux sommitaux de l'unité U2.

B) surface fortement bioturbée du banc carbonaté.

C) Les niveaux gréseux sommitaux de l'unité U2 affectés par des failles synsédimentaires.

Photo 4: Vue panoramique d'une partie de la coupe Est (1ère coupe), montrant la sous unité 2 silteuse et rougeâtre de U3, surmontée par la sous unité 1 de U4 qui forme une corniche carbonatée.

Photo 5 : Détail de la précédente figure, montrant la surface (SIM4) à nodules de silex de la sous unité 1 de U4.

\section{Planche 3}

Photo 1 : Banc calcaire lumachellique à Gastéropodes. (sous unité 1 de l'unité 3$)$.

Photo 2: Détail de la photo précédente.

Photo 3: Faille synsédimentaire à rejet métrique.

Photo 4 : Faille synsédimentaire à rejet centimétrique. 


\section{Planche1}
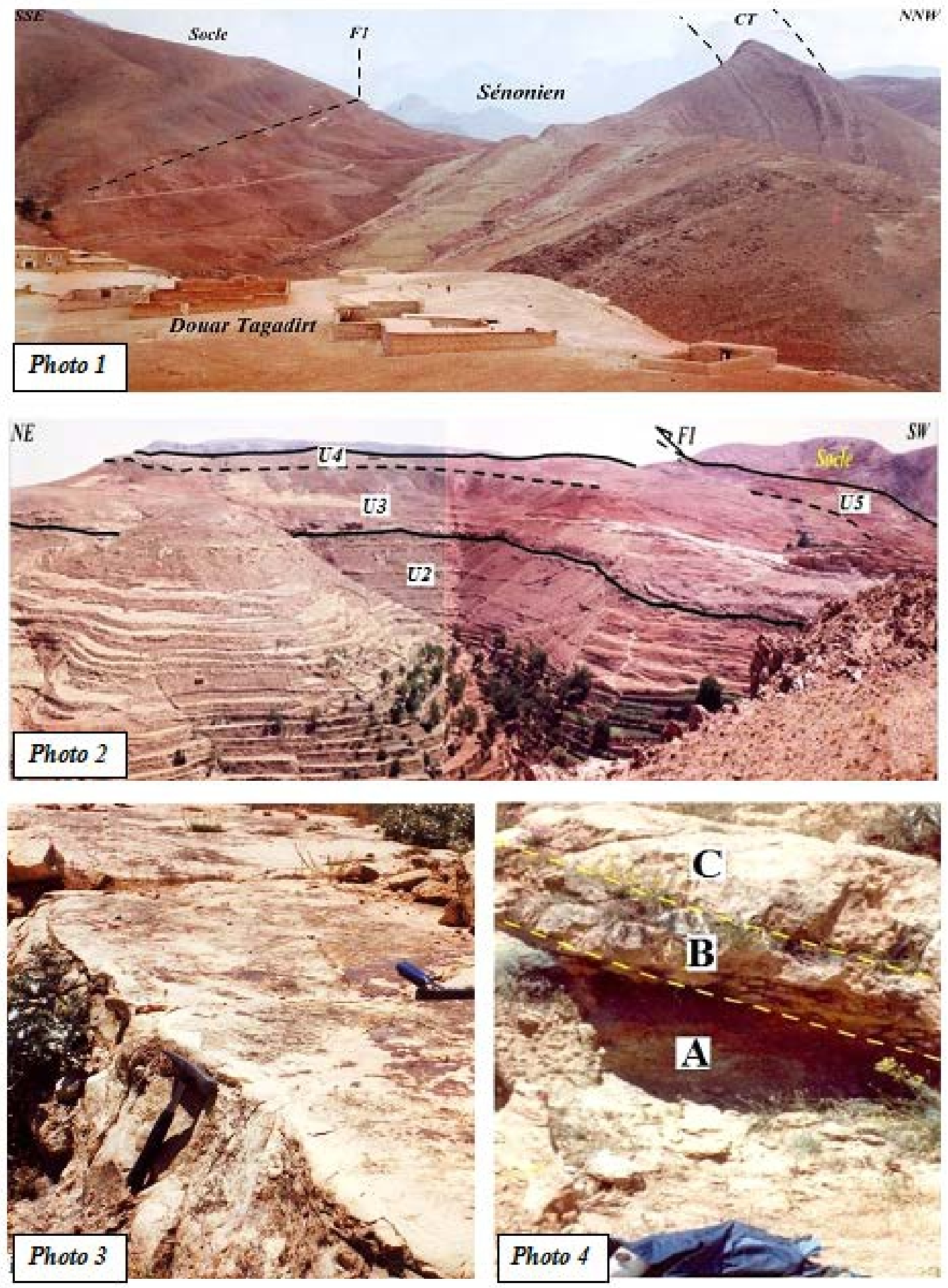


\section{Planche2}
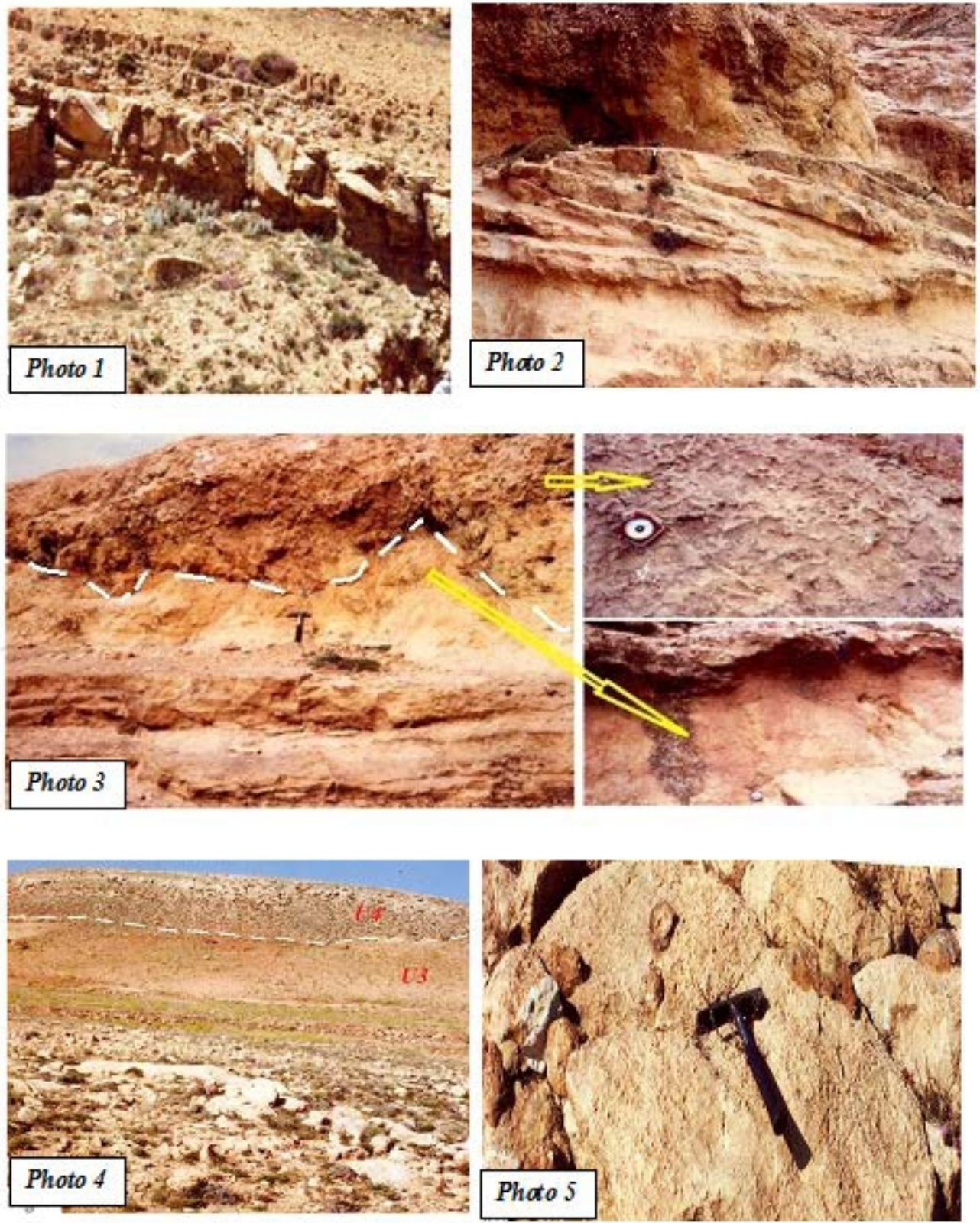


\section{Planche 3}
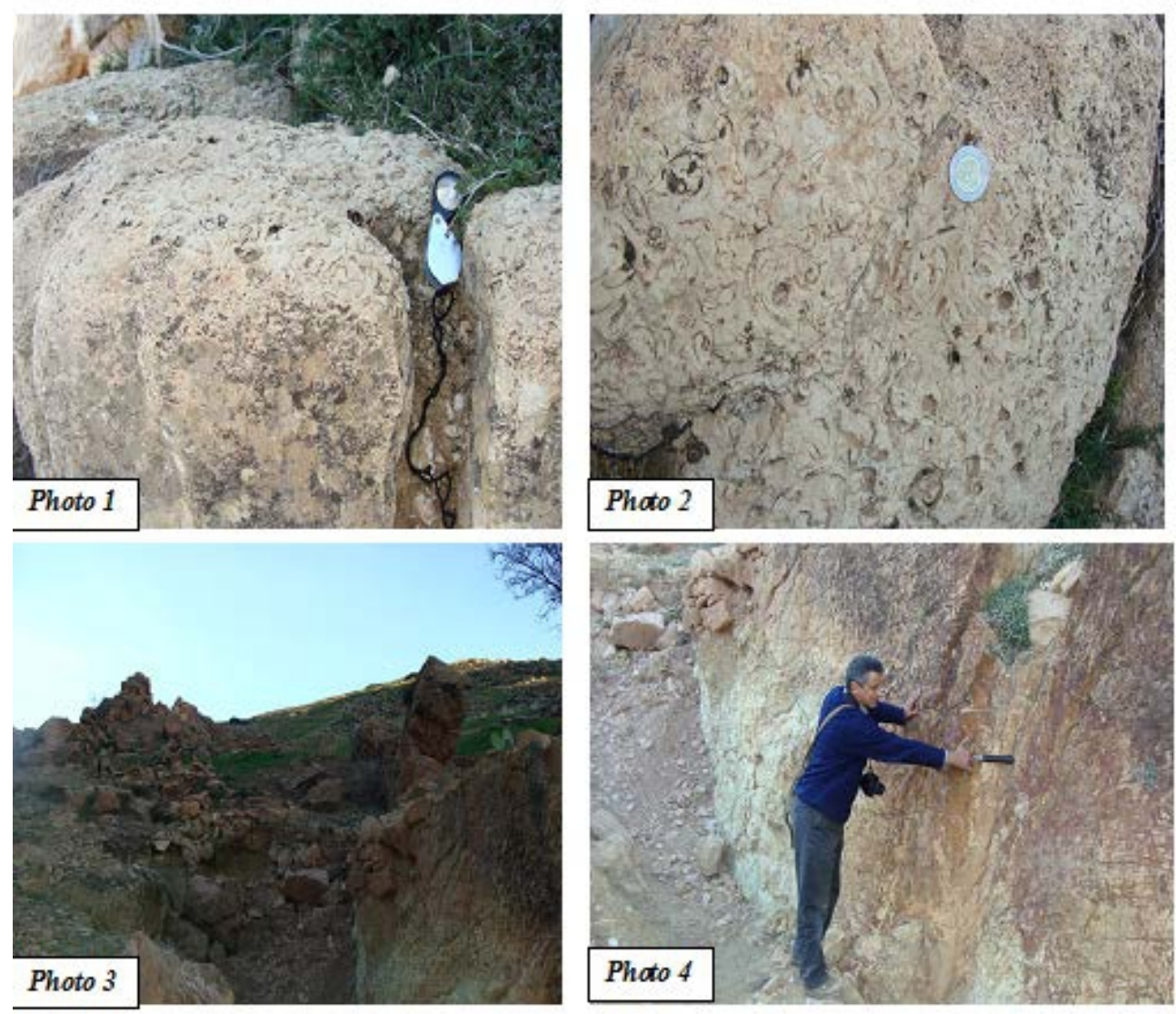

\section{References:}

ALGOUTI A. (1999). Le Sénonien (Coniacien, Santonien, Campanien et Maastrichtien) du Haut Atlas occidental (MAROC) : Sédimentologie, stratigraphie séquentielle, biostratigraphie et évolution géodynamique. Thèse d'Etat es-Science, Fac. Sci. Semlalia, Marrakech

ALGOUTI, A.; ALGOUTI, AB. et HADACH, F. (2015). Le Crétacé supérieur de la région d'Imin'Tanout (Haut Atlas Occidentel. Maroc) : Sédimentologie, Biostratigraphie et Analyse Séquentielle. European Scientific Journal (ESJ), Volume 11, N²4, pp.182-204.

BURRI et Al. (1973). Teepees structures and associated diagenitic features in intertidal carbonates sands (Lower Jurassic, Morocco.Sediment. Geol., 9 pp.813-823, Amsterdam. 
DRESNAY R. Du (1976). Signification paléoclimatique et paléogéographique de la présence de structures en "tipis", au Lias inférieur, dans le domaine des chaines atlasiques du Maroc. C. R. Acad. Sc. Paris, t.282, Série D, pp.2147-2149.

HADACH, F.; ALGOUTI, A.; ALGOUTI, AB. et MOURABIT, Z. (2015). Example of paleosebkha littoral deposits of Senonian in the "Basins Zone" of Aït Ourir (Marrakech High Atlas, Morocco). European Scientific Journal (ESJ), Volume11, N8, pp.306-316.

HADACH, F.; ALGOUTI, A. et ALGOUTI, AB. (2015). Etude sédimentologique du Trias et Jurassique du Haut Atlas de Marrakech, Maroc : Contribution à la localisation des niveaux favorables à l'exploitation artisanale pour la poterie. European Scientific Journal (ESJ), Volume 11, $\mathrm{N}^{\circ} 21$, pp.308-333.

PURSER B. H (1980). Sédimentation et diagenèse des carbonates néritiques récents. Technip. Paris, 1, 366p. 192fig 